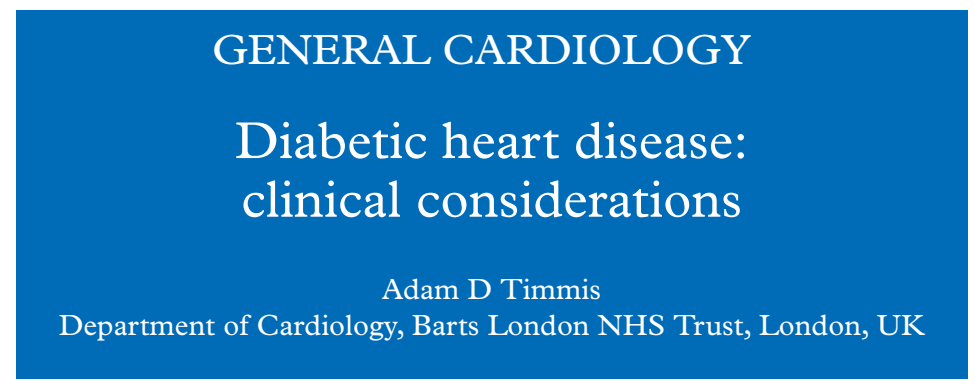

$\mathrm{D}$ iabetes is one of the most common metabolic disorders, and with our aging, sedentary and increasingly obese population, the number of affected individuals will continue to rise. This will have major implications for cardiological practice since much of the excess morbidity and mortality among diabetic patients is attributable to accelerated atherogenesis. Young diabetics are at particular risk and, by the age of 50 years, $33 \%$ of those requiring insulin have died from coronary heart disease. Indeed, $75 \%$ of all deaths in patients with diabetes are from this cause. Generally speaking, the management of diabetic patients with heart disease is underpinned by the same evidence base as applies to non-diabetic patients, and it is noteworthy that $15-20 \%$ of the patients in most of the landmark clinical trials have been diabetic. Recently, however, trials such as the UKPDS, HOPE, and DIGAMI studies (see below) have identified novel strategies for reducing cardiovascular risk in diabetes. These trials have already had a major impact on cardiological practice, emphasising the prime importance of blood pressure control and converting enzyme inhibition for reducing cardiovascular risk in diabetes, and also the value of insulin treatment for reducing mortality in diabetic myocardial infarction. Additional trials, already in progress, are expected to refine further the cardiovascular management of patients with diabetes in order to provide an effective challenge for a problem that shows no signs of going away.

\section{Diabetes and cardiovascular risk}

Three large epidemiological studies have shaped current understanding of the natural history of diabetic heart disease. The Framingham study showed that diabetes increased the relative risk of coronary heart disease by $66 \%$ in men and $203 \%$ in women followed up for 20 years, after controlling for the effects of age, smoking, blood pressure, and cholesterol. ${ }^{1}$ The Whitehall study of male civil servants extended these observations by showing that subclinical glucose intolerance, in addition to frank diabetes, also increased coronary risk. ${ }^{2}$ The MRFIT trial, with its very large population of middle aged men, was able to provide more detailed information about the interaction between diabetes and other risk factors in determining coronary risk. ${ }^{3}$ This trial confirmed the heightened risk attributable to diabetes, and also the independent effects of

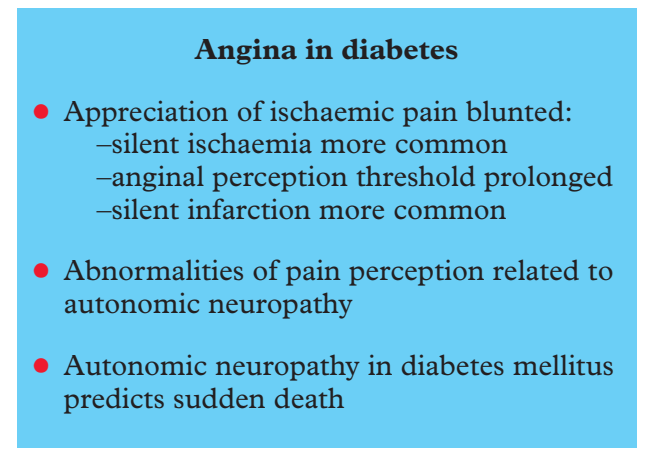

serum cholesterol, blood pressure, and smoking in men with and without diabetes. MRFIT showed that in men with diabetes, 12 year cardiovascular mortality was much higher at every level of these major risk factors considered singly and in combination, and that with progressively more unfavourable risk factor status the mortality rate rose much more steeply than in men without diabetes (fig 1).

\section{Angina and revascularisation in} diabetes

Angina may affect up to $40 \%$ of adults with diabetes, although its precise prevalence is hard to deduce from the literature. Symptoms are commonly atypical, perhaps because of abnormalities in the perception of angina caused by autonomic neuropathy, and the physician should retain a low threshold for non-invasive investigation. A positive stress test associated with atypical symptoms in the diabetic patient indicates a high probability of underlying coronary disease and the need for specific antianginal treatment, often with additional angiographic assessment. The disease is typically diffuse, affecting both proximal and distal coronary segments (angiographic observations not always borne out by pathological studies), and this has the potential to intensify ischaemia.

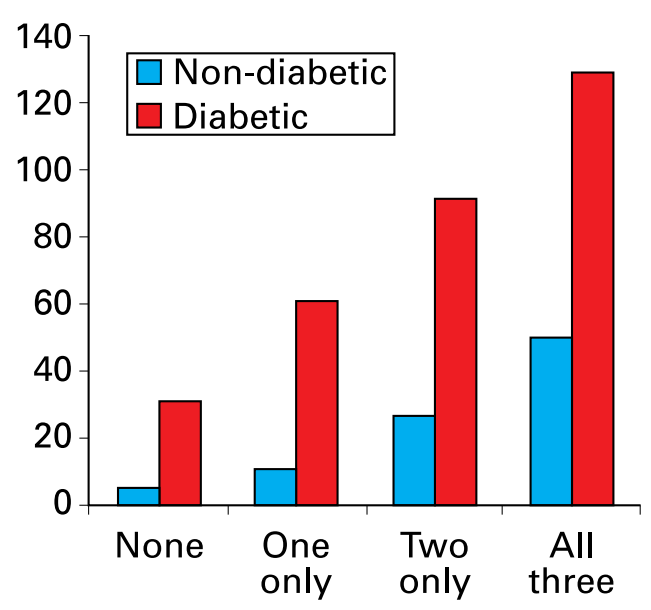

Figure 1. MRFIT trial: age adjusted cardiovascular disease death rates (per 10000 person years) by presence of number of risk factors in men with and without diabetes. Reproduced from Stamler et $\mathrm{al}^{3}$ with permission of American Diabetes Association. 


\section{Revascularisation in diabetes}

- Diffuse and distal disease makes revascularisation technically more demanding

Reduced survival after coronary artery bypass graft (CABG) and percutaneous transluminal coronary angioplasty (PTCA)

- Increased restenosis rates after PTCA

- BARI data indicate that for most diabetics CABG is preferable to PTCA

Recent EPISTENT data point to an important role for stenting and IIb/IIIa receptor blockers in diabetic patients undergoing PTCA

There is no evidence of reduced responsiveness to medical treatment in patients with diabetes, which should be with nitrates and $\beta$ blockers in the first instance with the addition of calcium channel blockers or potassium channel openers in more resistant cases. However, diffuse disease makes revascularisation by angioplasty or bypass surgery more difficult and more hazardous. Indeed, diabetes has long been recognised as one of the major independent predictors of long term mortality after surgery. The results of angioplasty also tend to be less good in diabetic compared with nondiabetic patients. Again, diffuse disease makes for technically more difficult angioplasty procedures and, in addition, restenosis rates are consistently higher. In the recent BARI trial of angioplasty versus bypass surgery, subgroup analysis showed that patients with diabetes randomised to angioplasty had a significantly worse five year survival than those randomised to bypass surgery. The investigators concluded that for most diabetics requiring revascularisation coronary bypass surgery was preferable to angioplasty. ${ }^{4}$ Nevertheless, BARI antedated the stenting era and recently a predefined subgroup analysis from the EPISTENT trial showed that angioplasty and stenting combined with infusion of abciximab (a glycoprotein IIb/IIIa receptor inhibitor) improves the long term outcome in diabetic patients substantially, with a six month incidence of ischaemic end points comparable to that achieved in non-diabetic patients. ${ }^{5}$ The data suggest, therefore, that stenting and IIb/IIIa receptor blockade may have an important role in diabetic angioplasty.

\section{Acute myocardial infarction in} diabetes

Framingham data have shown that the risk of acute myocardial infarction is $50 \%$ greater in diabetic men and $150 \%$ greater in diabetic women than in non-diabetic individuals. ${ }^{1}$ Indeed, acute myocardial infarction accounts for $30 \%$ of all diabetic deaths. This propensity to myocardial infarction presumably reflects the increased prevalence of coronary artery disease in diabetes, with associated hypertension predisposing to plaque rupture. Moreover, thrombotic responses to plaque rupture are likely to be exaggerated in diabetes because of haematological abnormalities, particularly increased platelet activation.

It has long been recognised that diabetics are prone to "silent" myocardial infarction and this presumably reflects impaired perception of ischaemic cardiac pain caused by autonomic neuropathy. Thus, in diabetes, acute myocardial infarction is silent or presents with atypical symptoms in $32-42 \%$ of cases compared with $6-15 \%$ of non-diabetic infarcts. This is disadvantageous because it has the potential to delay access to emergency facilities early after coronary events, increasing the risk of out-of-hospital sudden death and morbid complications of myocardial infarction, particularly cardiogenic shock. Abnormalities of circadian and seasonal rhythms of acute myocardial infarction, with attenuation of the morning and winter peaks, may also reflect autonomic dysfunction because these rhythms are largely driven by parallel changes in sympathovagal activity. The consequences of this to the patient with diabetes are unclear.

All the major complications of myocardial infarction occur more commonly in diabetes, particularly heart failure which affects nearly $50 \%$ of diabetics compared with under $30 \%$ of non-diabetics (fig 2). This difference is not accounted for by infarct size but may reflect the more severe and diffuse disease in diabetes that limits coronary reserve and intensifies ischaemia in non-infarcted segments by a watershed effect. ${ }^{6}$ Diabetes specific myocardial disease may also have a role. Thus, contractile dysfunction remote from the infarct zone is commonly reported in diabetic myocardial infarction. ${ }^{6}$ Since heart failure is one of the major determinants of outcome, it is little surprise that both hospital and long term mortality rates are increased in patients with diabetes. In our own coronary care unit, the 30 day and 12 month mortality rates $(95 \%$ confidence intervals) for patients with diabetes are $19.2 \%$ $(15.3 \%$ to $23.1 \%)$ and $26.6 \%(22.2 \%$ to $31.1 \%)$, compared with $12.7 \%(10.6 \%$ to $14.8 \%)$ and $19.1 \%(16.6 \%$ to $21.6 \%)$ for patients without diabetes.

Patients with acute myocardial infarction who have diabetes, or an admission blood glucose concentration $\leqslant 11.0 \mathrm{mmol} / \mathrm{l}$, should receive insulin and glucose infusion for at least 24 hours based on the findings of the DIGAMI investigators who showed that this significantly improves survival. ${ }^{7}$ Subcutaneous insulin treatment continued for at least three months in this study, a regimen that many units find too unwieldy to apply in practice, largely because of the unwillingness of many patients with mild diabetes to self inject. Certainly treatment during the acute phase of infarction is likely to be particularly beneficial, not only for improving left ventricular function, but also for reducing infarct size and lethal complications, since the shift to anaerobic glucose metabolism that occurs in the myocardium during acute ischaemia is an insulin 


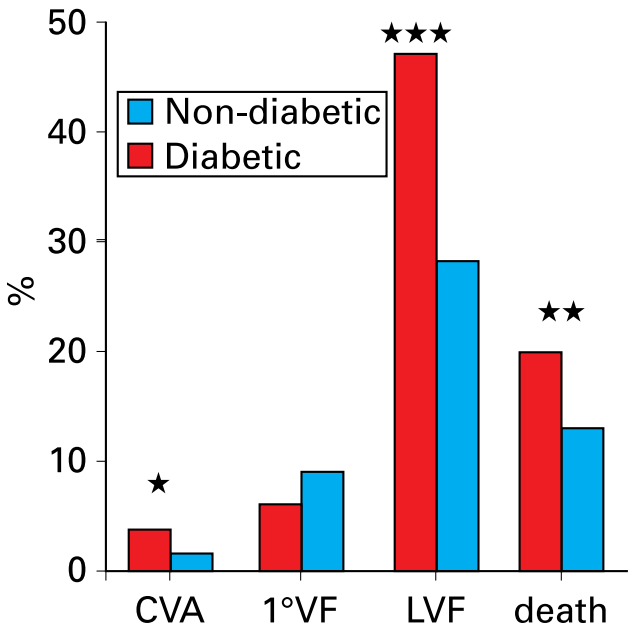

Figure 2. Complications of acute myocardial infarction in diabetic and non-diabetic patients $(n=$ 1929). CVA, cerebrovascular accident; $1^{\circ} \mathrm{VF}$, primary ventricular fibrillation; LVF, left ventricular failure. ${ }^{*} p<0.05 ;{ }^{* *} p<0.01 ;{ }^{* * *} p<0.001$. Unpublished data from the Newham General Hospital coronary care unit database.

dependent adjustment that may be deficient in diabetes because of absolute or relative lack of insulin. ${ }^{8}$

In other respects the treatment of myocardial infarction in patients with diabetes should be the same as in those without-responses to thrombolytic treatment and the benefits of secondary prevention being similar in the two groups. The TAMI investigators, for example, reported that patency rates of the infarct related artery 90 minutes after thrombolytic treatment were almost identical in patients with and without diabetes. ${ }^{6}$ Similarly, diabetes does not appear to affect the benefits of aspirin, or $\beta$ blockers and statins which provide protection against recurrent infarction and death comparable to that seen in patients without diabetes. Angiotensin converting enzyme (ACE) inhibitors, in particular, have a special role and should be given to all diabetic patients with acute myocardial infarction, not only because of the heightened risk of left ventricular failure, for which these drugs are of proven benefit, but also because of the protection they afford against microvascular complications.

\section{Sudden death in diabetes}

Patients with diabetes are at increased risk of sudden death, and this is not always attributable to complications of plaque events. There is considerable evidence that autonomic neuropathy plays an important pathophysiological role, through prolongation of QT interval and selective reductions in vagal function (increasing sympathetic activity), both of which may increase susceptibility to lethal arrhythmias. ${ }^{9} 10$ In addition, altered perception of ischaemic cardiac pain may deprive diabetic patients of the signal to stop exercising, allowing ischaemia to intensify to the point that arrhythmias are triggered. ${ }^{11}$ Alterations in pain perception may also prevent the diabetic patient from seeking medical attention in the event of acute coronary syndromes or lead to inappropriate triage decisions in the emergency room, such that access to defibrillators is denied and specific treatment delayed. This emphasises the importance of retaining low diagnostic thresholds for coronary heart disease in the diabetic patient presenting with atypical symptoms. It also emphasises the importance of strict glycaemic control in diabetes, although whether this indeed protects against neuropathy is unclear.

\section{Heart failure in diabetes}

Over 20 years ago, the Framingham investigators reported that the annual incidence of heart failure in diabetic men and women was substantially greater across all age groups than in non-diabetic individuals, even after controlling for underlying coronary and rheumatic heart disease. They concluded that diabetes itself might predispose to heart failure independently of concurrent coronary or rheumatic heart disease. At about the same time postmortem reports appeared on diabetics with heart failure describing normal coronary arteries and heart valves. Histology, however, may reveal a range of abnormalities including myocyte hypertrophy, interstitial fibrosis, increased periodic acid-Schiff (PAS) positive material, and intramyocardial microangiopathy. These are largely indistinguishable from changes found in hypertensive left ventricular disease and emphasise the importance of effective antihypertensive treatment as reported in UKPDS (see below). In further studies, analysis of systolic time intervals provided evidence of both systolic and diastolic left ventricular dysfunction in diabetic individuals in whom there was no clinical evidence of coronary artery disease. Taken together these epidemiological, pathological, and haemodynamic data provide the evidence base for diabetes specific myocardial disease, commonly called "diabetic cardiomyopathy". The pathogenesis is unclear, although possible mechanisms include the synergistic impact of hypertension plus chronic derangement of myocardial metabolism, with increased free fatty acid oxidation and decreased glucose utilisation. ${ }^{12}$

While the existence and clinical importance of diabetic cardiomyopathy is fairly well established, it must be emphasised that coronary heart disease is considerably more important as a cause of heart failure in the patient with diabetes. Certainly some of the epidemiological data summarised above may have been distorted by coexisting but asymptomatic coronary heart disease, and the pathological data do not rule out the possibility of cardiomyopathy as a coincidental diagnosis or as a response to hypertension. In practical terms, however, the distinction is largely unimportant, except insofar as it affects revascularisation decisions, since treatment strategies are unaffected and are the same as for non-diabetic patients with heart failure. Thus, control of provocative factors, particularly arrhythmias and hypertension, 


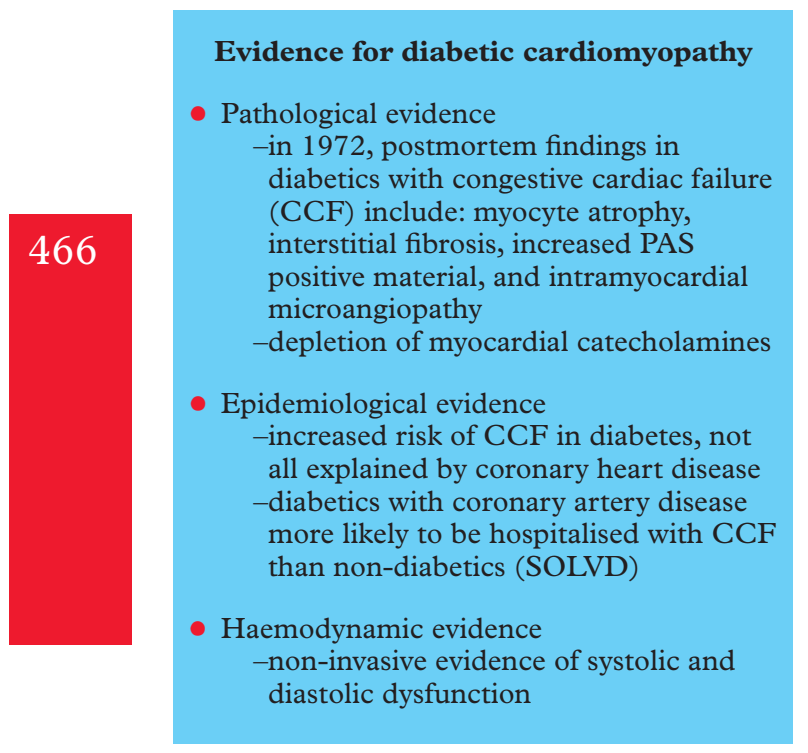

remains essential and conventional treatment should be applied for correcting fluid retention, increasing exercise capacity, and improving prognosis. Diuretics may adversely influence metabolic control in diabetes but are mandatory for treatment of peripheral oedema and pulmonary congestion. The efficacy of ACE inhibition is undiminished in diabetes, judging by subgroup analyses of the SOLVD prevention and treatment trials, but the heightened risk of renal artery stenosis demands caution when initiating treatment. $\beta$ Blockers are also recommended, although this is based on generalisation from randomised trials rather than specific data for patients with diabetes.

Risk factor modification for protecting against coronary heart disease in diabetes

Based on their data, the MRFIT investigators recommended "vigorous sustained intervention in people with diabetes to control blood pressure, lower serum cholesterol, and abolish cigarette smoking". These recommendations remain central to the cardiovascular management of diabetes, and their value for primary and secondary prevention has been confirmed in randomised trials. Disappointingly, however, there is not yet clear evidence that the recommendation for more vigorous risk factor modification in diabetes leads to extra protection against coronary heart disease beyond that achieved in non-diabetic individuals, although important protection against microvascular complications (retinopathy, renal disease) does occur. Nevertheless, as our practice evolves from single to multifactorial risk assessment, in which an individual's absolute coronary risk can be readily assessed from simple colour coded charts, the clinical impact of risk factor modification can be expected to increase, particularly with the recent availability of charts for use in men and women with diabetes.
Clinical end point

Any diabetes related end point

Deaths related to diabetes

All cause mortality

Myocardial infarction

Stroke

Peripheral vascular disease

Microvascular disease

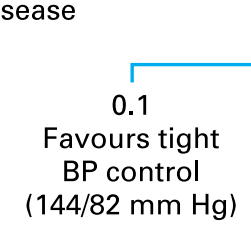

Relative risk (95\% Cl)

$0.76 \quad(0.62$ to 0.92$)$

$0.68 \quad(0.49$ to 0.94$)$

$0.82 \quad(0.63$ to 1.08$)$

$0.79 \quad(0.59$ to 1.07$)$

$0.56 \quad(0.35$ to 0.89$)$

$0.51 \quad(0.19$ to 1.37$)$

$0.63 \quad(0.44$ to 0.89$)$

10

Favours less tight BP control

(154/87 mm Hg)

Figure 3. UKPDS 38 trial: relative risk reduction with tight blood pressure control. ${ }^{13}$

\section{Blood pressure}

Hypertension commonly occurs in type 2 diabetes, and contributes importantly to the heightened risk of cardiovascular, renal, and retinal disease. By the age of 50 years more than $40 \%$ of patients with type 2 diabetes are hypertensive, the proportion rising to $60 \%$ by the age of 75 years. Subgroup analyses of large trials have suggested that the benefits of treating hypertension apply equally to diabetic and non-diabetic patients, a suggestion emphatically confirmed in the hypertensive cohort of UKPDS. ${ }^{13}$ Comparison of patients allocated either to tight blood pressure control $(<150 /$ $85 \mathrm{~mm} \mathrm{Hg}$ ) using captopril or atenolol, or to less tight control showed that tight control for 8.4 years was associated with significant reduction in the risk of death related to diabetes, and with reductions in all microvascular endpoints. Predictably, reductions in the risk of heart failure and stroke also occurred, but the $21 \%$ reduction in the risk of myocardial infarction was not significant (fig 3). The HOT study also reported reductions in myocardial infarction in patients treated to a target diastolic blood pressure of $\leqslant 80 \mathrm{~mm} \mathrm{Hg}$ compared to targets of $\leqslant$ 85 or $\leqslant 90 \mathrm{~mm} \mathrm{Hg}$, but again the changes were not significant. Based largely on these recent trial data, a target blood pressure of $<130 \mathrm{~mm} \mathrm{Hg}$ systolic and $<80 \mathrm{~mm} \mathrm{Hg}$ diastolic is now recommended for diabetic patients. Lower targets might be appropriate for diabetic patients with microalbuminuria, in whom considerable data support the use of ACE inhibitors for protecting against deterioration of renal function, a beneficial effect that occurs independently of blood pressure reduction. However, UKPDS reported that captopril or atenolol were similarly effective in reducing the incidence of diabetic complications, and concluded that for most patients blood pressure reduction itself is more important than the agent used. ${ }^{14}$

Lipid modification with fibrates and statins Hypertriglyceridaemia with reductions in high density lipoprotein (HDL) cholesterol are the 
typical abnormalities detected on routine laboratory testing in type 2 diabetes. This provides a logic for fibrate treatment in addition to exercise and weight reduction. The Helsinki study suggested a trend towards reduced coronary events in diabetic patients treated with gemfibrozil for five years, but data from other fibrate studies have generally been inconclusive. This contrasts with subgroup analyses of the major statin trials which have shown convincingly that hypercholesterolaemic diabetic patients gain similar relative benefit as non-diabetic patients in the secondary prevention of coronary artery disease, and greater absolute benefit because of their higher event rate. Thus, vigorous treatment of all diabetics with known atherosclerotic disease to achieve a total cholesterol of $<5.0 \mathrm{mmol} / 1$ (low density lipoprotein (LDL) cholesterol $<3.0 \mathrm{mmol} / \mathrm{l}$ ) is recommended, together with correction of hypertriglyceridaemia using additional fibrate treatment, as necessary, In diabetic patients without overt atherosclerotic disease, an absolute risk $\geqslant 30 \%$ of developing coronary heart disease over the next 10 years, as deduced from colour coded risk prediction charts, is sufficiently high to justify drug treatment.

\section{Smoking}

Observational data suggest that the risk of myocardial infarction is reduced by up to $50 \%$ within one year of quitting smoking, with favourable effects on mortality maintained for up to 10 years. Since the cardiac risk attributable to smoking is magnified considerably in diabetes, as indeed is the risk attributable to all other risk factors, the benefits of quitting are likely to be as great, if not greater, in diabetic then non-diabetic patients.

\section{Glycaemic control for protecting against coronary heart disease in diabetes}

Strict glycaemic control has long been recommended in diabetes, based on epidemiological surveys that have reported more favourable clinical outcomes for groups with lower plasma glucose and glycosylated haemoglobin concentrations. However, whether these more favourable outcomes reflected less severe underlying disease rather than the benefits of glycaemic control remained unresolved until publication of UKPDS in which 3867 newly diagnosed patients with type 2 diabetes were randomly assigned to an intensive (sulfonylurea or insulin) or conventional treatment policy. ${ }^{15}$ After follow up for 10 years, glycosylated haemoglobin concentrations in the two groups were $7.0 \%$ and $7.9 \%$, respectively, a difference of only $11 \%$. Nevertheless, this trial confirmed the close relation between glycaemia and the risk of microvascular and macrovascular complications, including coronary heart disease, and also dispelled concerns about the potential adverse cardiovascular effects of sulfonylureas. Importantly, in the group randomised to intense glycaemic control, significant protec- tion against microvascular complications occurred although macrovascular complications were not similarly affected, the $16 \%$ reduction in the risk of myocardial infarction being of only borderline significance. In short, therefore, UKPDS has confirmed the importance of strict glycaemic control (glycosylated haemoglobin $7 \%$ or lower) for protection against microvascular complications of diabetes. It is tempting to speculate that more substantial protection against macrovascular end points might have occurred had there been greater differences in glycaemic control (as reflected by glycosylated haemoglobin) between the intensive and conventional treatment groups.

Other strategies for protecting against coronary heart disease in diabetes

Antiplatelet treatment

An overview of randomised trials has shown that the benefits of antiplatelet treatment for secondary prevention of coronary heart disease are similar for groups with and without diabetes. Thus patients with diabetic coronary heart disease should all receive a daily aspirin. Though not strictly evidence based, aspirin is now recommended for diabetic adults without clinical manifestations of atheromatous disease (primary prevention) since platelet dysfunction is common and the prevalence of subclinical disease high. Evidence for non-aspirin platelet inhibitors in diabetic subgroups is often unavailable, but it is reasonable to assume that the potential benefits of glycoprotein IIb/IIIa receptor antagonists in non-ST elevation coronary syndromes apply equally to patients with diabetes. At present the proven benefit of these drugs is largely restricted to the catheter laboratory where they have a vital role in high risk angioplasty, particularly as an adjunct to coronary stenting, ${ }^{5}$ reducing the rate of adverse events in patients with diabetes to a level comparable to that of patients without diabetes (see above).

\section{ACE inhibition}

ACE inhibition protects against the development of atherosclerotic plaque in experimental animals fed lipid rich diets. Potential for similar benefit in humans was reported by the TREND investigators who showed that treatment with quinapril improved coronary endothelial function in patients with coronary disease. ${ }^{16}$ This potential has now been confirmed by the HOPE study in which significant reductions in the risk of the combined primary outcome (death, myocardial infarction, and stroke) occurred in high risk patients randomised to treatment with ramipril. ${ }^{17}$ Among these high risk patients were 3577 with diabetes who had a previous cardiac event or at least one other cardiovascular risk factor, but not heart failure or proteinuria. Within this diabetic subgroup, randomisation to ramipril reduced the risk of the combined primary outcome by $25 \%$, with an additional reduction in the risk of overt nephropathy. ${ }^{18}$ These important findings are 


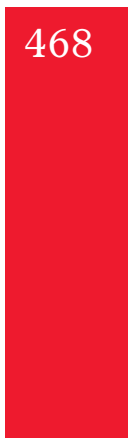

Trial acronyms
BARI: $\begin{gathered}\text { Bypass Angioplasty } \\ \text { Revascularization Investigation }\end{gathered}$
DIGAMI: Diabetes Mellitus, Insulin
Glucose Infusion in Acute
Myocardial Infarction

now having a major impact on the management of diabetes, providing indications for ACE inhibition with ramipril in any diabetic patient with multiple risk factors, established vascular disease, or microalbuminuria.

\section{Screening for coronary heart disease in diabetes}

The prevalence of subclinical coronary artery disease in the general population is high, but is almost certainly higher in the diabetic population because of accelerated atherosclerosis. Thus, people with diabetes have a long term rate of myocardial infarction and cardiovascular death comparable to that of non-diabetic patients with a documented history of myocardial infarction. Subclinical disease is commonly non-obstructive because of outward remodelling of the coronary artery. However, obstructive disease may also be clinically silent, particularly in diabetes when autonomic neuropathy may interfere with the perception of cardiac pain such that symptoms take longer to develop after the onset of myocardial ischaemia (prolonged anginal perceptual threshold) or do not occur at all (silent ischaemia).

The relatively high prevalence of subclinical coronary artery disease associated with diabetes has led to debate about the value of screening programmes using non-invasive tests. As a universal principal, this can scarcely be justified, the sensitivity of stress testing (electrocardiographic or perfusion imaging) for detecting subclinical disease being low with only a $5-10 \%$ incidence of obstructive lesions ( $>50 \%$ luminal narrowing at angiography) among asymptomatic diabetic cohorts. Moreover, the mere demonstration of obstructive coronary disease does not usually affect ongoing management with strict glycaemic control and risk factor modification. Certainly, there is no evidence to support angioplasty in asymptomatic cases, while the potential prognostic benefits of surgery in the minority with three vessel or left main disease needs to be balanced against the heightened procedural risk and less favourable longer term outcome in patients with diabetes. Nevertheless, in certain subgroups, screening for coronary artery disease is recommended because it can lead to treatment strategies that favourably affect prognosis. These include diabetic patients needing renal transplantation or major non-cardiac vascular surgery in whom coronary revascularisation may reduce the procedural risk.

1. Kannel WB, McGee DL. Diabetes and cardiovascular risk factors: the Framingham study. Circulation

1979;59:8-13.

- Subset analysis from the father of all epidemiological studies.

2. Fuller JH, Shipley MJ, Rose G, et al. Mortality from coronary heart disease and stroke in relation to degree of glycaemia: the Whitehall study. BMJ 1983;287:867-70.

- The classic Whitehall study which for the first time related the risk of stroke, as well as coronary heart disease, to the degree of ischaemia.

3. Stamler J, Vaccaro O, Neaton J, et al. Diabetes, other risk factors, and 12-yr cardiovascular mortality for men screened in the multiple risk factor intervention trial. Diabetes Care 1993;16:434-44.

- The massive MRFIT study confirmed the independent risk attributable to diabetes over and above the effect of smoking, lipids and blood pressure.

\section{The Bypass Angioplasty Revascularization} Investigation (BARI) Investigators. Comparison of coronary bypass surgery with angioplasty in patients with multivessel disease. N Engl J Med 1996;335:217-25.

- The randomised trial that called into question the use of angioplasty for revascularising diabetic patients with coronary artery disease.

5. Marso SP, Lincoff AM, Ellis SG, et al. Optimizing the percutaneous interventional outcomes for patients with diabetes mellitus: results of the EPISTENT (Evaluation of platelet Ilb/IIla inhibitor for stenting trial) diabetic substudy. Circulation 1999;100:2477-84.

- This study produced a sigh of relief from interventionists by showing that stenting plus abciximab in diabetics produces results similar to those achieved in non-diabetic patients, justifying percutaneous revascularisation in this patients, justifying
high risk group.

6. Granger CB, Califf RM, Young S, et al, and The Thrombolysis and Angioplasty in Myocardial Infarction (TAMI) Study Group. Outcome of patients with diabetes mellitus and acute myocardial infarction treated with thrombolytic agents. J Am Coll Cardiol 1993;21:920-5 - An influential study confirming the equal benefits of thrombolytic treatment for achieving coronary patency in diabetic and non-diabetic patients with acute myocardial infarction.

7. Malmberg K, Ryden L, Efendic S, et al for the Diabetes Mellitus, Insulin Glucose Infusion in Acute Myocardial Infarction (DIGAMI) Study Group. Randomized trial of insulin-glucose infusion followed by subcutaneous insulin treatment in diabetic patients with acute myocardial infarction: effects on mortality at 1 year. J Am Coll Cardiol 1995;26:57-65.

- One of those studies that had a major impact on clinical practice. Since its publication glucose and insulin infusion has become recommended practice for all diabetic patients with acute myocardial infarction.

8. McGuire DK, Granger CB. Diabetes and ischemic heart disease. Am Heart J 1999;138:S336-75.

- State of the art review of diabetic coronary heart disease.

9. Ewing D, Boland O, Neilson J, et al. Autonomic neuropathy, QT interval lengthening, and unexpected deaths in male diabetic patients. Diabetologia 1991;34:182-5.

- One of several classic papers from Ewing highlighting the One of several classic papers from Ewing highlighting the
important interaction between autonomic neuropathy and clinical manifestations of coronary heart disease in diabetes.

10. Marchant B, Umachandran V, Stevenson R, et al. Silent myocardial ischaemia: the role of subclinical neuropathy in patients with and without diabetes. J Am Coll Cardiol 1993;22:1433-7.

- Debate about the relation between diabetes and silent Debate about the relation between diabetes and silent
ischaemia was resolved by this study which showed that silent ischaemia was largely confined to neuropathic subsets.

11. Ranjadayalan K, Umachandran V, Ambepityia G, et al. Prolonged anginal perceptual threshold in diabetes: effects on exercise capacity and myocardial ischemia. J Am effects on exercise capacity and
Coll Cardiol 1990;16:1120-4.

Coll Cardiol 1990;16:1120-4.
- This study showed how impaired perception of ischaemic This study showed how impaired perception of ischaemic
cardiac pain in patients with diabetes deprives them of the signal to stop exercising as ischaemia intensifies. 
12. Solang L, Malmberg K, Ryden L. Diabetes mellitus and congestive heart failure: further knowledge needed. Eur Heart J 1998:20:789-95.

- An authoritative review of heart failure as it affects patients with diabetes.

13. United Kingdom Prospective Diabetes Study (UKPDS) Group. Tight blood pressure control and risk of diabetes (UKPDS 38). BMJ 1998;317:703-13.

diabetes (UKPDS 38). BMJ 1998;317:703-13. protective effects of tight blood pressure control.

14. United Kingdom Prospective Diabetes Study (UKPDS) Group. Efficacy of atenolol and captopril in reducing risk of maro educing

1998;317:713-20.

- This study raised a few eyebrows when it reported that ACE inhibitors offered no apparent advantage over $\beta$ blockers for protecting against microvascular complications in hypertensive diabetics.

15. United Kingdom Prospective Diabetes Study (UKPDS) Group. Intensive blood-glucose control with
sulphonylureas or insulin compared with conventional sulphonylureas or insulin compared with conventional
treatment and risk of complications in patients with type 2 diabetes (UKPDS 33). Lancet 1998;352:837-53.

- Another landmark publication from the UKPDS

investigators confirming unequivocally the benefits of strict glycaemic control for protecting against microvascular complications of diabetes.

16. Mancini GB, Henry GC, Macaya C, et al.

Angiotensin-converting enzyme inhibition with quinapril improves endothelial vasomotor dysfunction in patients with coronary artery disease. The TREND (trial on reversing

endothelial dysfunction) study. Circulation 1996;94:258-65.

- The first study in humans to show that ACE inhibition improves endothelial function in diseased coronary arteries.

17. Yusuf $S$, Sleight $P$, Pogue J, et al, for The Heart Outcomes Prevention Evaluation (HOPE) Study Investigators. Effects of an angiotensin-converting-enzyme inhibitor, ramipril, on cardiovascular events in high-risk

patients. N Engl J Med 2000;342:145-53.

- A landmark study confirming the benefits of ACE inhibition for protecting against ischaemic events in high risk patients.

18. Heart Outcomes Prevention Evaluation (HOPE) Study Investigators. Effects of ramipril on cardiovascular and microvascular outcomes in people with diabetes mellitus: result of HOPE study and MICRO-HOPE substudy. Lancet 2000;355:253-9.

- A crucial substudy of HOPE showing the benefits of ACE inhibition in diabetic patients for protecting against microvascular and macrovascular end points. 